\title{
Visual discrimination versus visuo-motor learning in interocular transfer in the fish'
}

EVELYN LEE-TENG

DIVISION OF BIOLOGY, CALIFORNIA INSTITUTE OF TECHNOLOGY

The hypothesis that interocular transfer in fish occurs in passive visual discrimination (PD), but not in visuo-motor learning (VM), was tested with 19 goldfish. An opaque eye cap was fitted on one eye before training, and later shifted to the other eye for the transfer test. In the VM problem, Ss had to learn new swimming patterns according to visual cues. The following PD problem involved a new pair of cues, but required the same swimming patterns. Results confirmed the hypothesis. Learning scores for each eye were comparable in the VM problem, but learning with the second eye was much faster in the PD problem.

Despite complete crossing of the optic nerves, interocular transfer (IT) of learning has been demonstrated with several kinds of fish (Ingle, 1965; Schulte, 1957; Sharpiro, 1965; Sperry \& Clark, 1949).However, lack of IT was found in goldfish when opaque eye caps were used to effect monocular vision and new swimming patterns were involved in the learning (McCleary, 1960). From this finding and some other supporting evidence, McCleary proposed that in fish IT occurs in passive visual discrimination (PD), but not in visuo-motor learning (VM) involving visually guided voluntary motor responses. This proposal fits the findings of the other studies where IT was demonstrated, because the motor responses involved were either the usual swim-to-food response, or were pretrained for both eyes. On the other hand, McCleary's evidence of lack of IT in VM was weak, as he only compared performance in a few beginning trials through the second eye with criterion performance through the first eye.

The present study further tested, with a different method, the hypothesis that in fish IT occurs in PD but not in VM. Ss were trained monocularly and tested for IT on each of two successive problems. The first problem involved easily discriminable visual cues but new visually guided swimming patterns. The second problem required the same motor performance with a new pair of discriminative cues. The hypothesis would predict lack of IT in the first problem, but presence of IT in the second problem. Training was carried to criterion level for each eye. Thus not only immediate IT, but also savings in learning, if any, will be revealed. Method

Nineteen goldfish, Carassius auratus, were individually trained, one eye at a time, in a "shuttle-box" situation. The Ss were taught to remain in one side or swim to the other side, depending on visual cues. Opaque eye caps molded from thin black acetate sheets were used to effect monocular vision. Each insertion or removal of the cap was performed while the $\mathrm{S}$ was under brief anesthesia by M.S.222. The apparatus is illustrated in Fig. 1. The enclosure was $4 \times 10 \times 6$ in. in height. Its two end walls, made of stainless steel, also served as electrodes for shock currents. The long side walls were made of white Plexiglas. A gray Plexiglas barrier with two 1-sq.-in. openings on its center piece divided the enclosure into two sides. Two pairs of stimulus patterns that served as the discriminative cues were used in two successive problems. The base for each pattern was a piece of stainless steel of approximately the same size as the end walls of the enclosure. In the first pair, the "positive" pattern was just a blank piece of untreated metal. The "negative" patterns had many $3 / 16$ in. red dots painted on the metal base. In the second pair, the positive pattern was white diagonal stripes, and the negative pattern black diagonal stripes. The stripes were each $3 / 8$ in. wide, with $1 / 4$ in. space in between. A transparent bar connected the two patterns of each pair at the middle of their top edge. When the pair was put into the enclosure, the patterns stood just in front of the end walls.

Each $\mathrm{S}$ had two daily training sessions, one in the morning, one in the afternoon. Eight "crossing" and eight "stay" trials were presented randomly in each session. For a "crossing" trial, the negative pattern was presented on S's side. The $S$ had $9 \mathrm{sec}$. to swim to the other side to avoid a maximum of three shocks. Each shock current was $0.2 \mathrm{sec}$. long, and the interval between two shocks was $7 \mathrm{sec}$. The intensity was about 5 ma. If the $S$ failed to swim to the other side after the three shocks, it was gently guided to the other side by the $\mathrm{E}$ with the use of a glass rod. In a "stay" trial, the positive patterns were presented at the S's side. If the $S$ remained

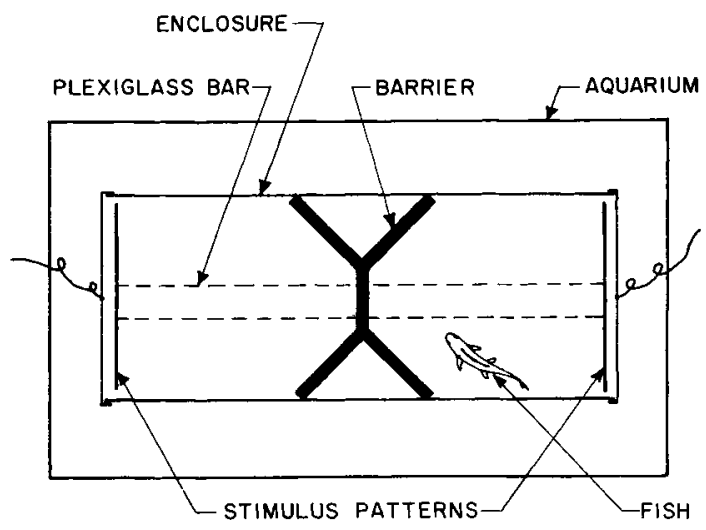

Fig. 1. Top view of the training apparatus. 
